\title{
Manasement of Paediatric Second-degree Burn in a Developing Country: With or Without Closed Dressing?
}

\author{
Md. Saif Ullah' \\ Kazi Md. Noor-ul Ferdous ${ }^{*}$ \\ Md. Mahboob Haider ${ }^{1}$ \\ Md. Khurshid Alam Sarwar ${ }^{2}$ \\ Muhd. Rashedul Alam ${ }^{1}$ \\ A.R. Khan ${ }^{1}$ \\ 'Department of Paediatric Surgery, \\ Dhaka Shishu (Children) Hospital \\ Dhaka, Bangladesh \\ ${ }^{2}$ Department of Paediatric Surgery, \\ Chittagong Medical College \& \\ Hospital, Chittagong, Bangladesh
}

\begin{abstract}
Objective: Our objective was to observe whether second-degree burn wound management can be done without using any occlusive dressing than conventional occlusive dressing in children. The purpose of this study was to evaluate our experience and to see the results of open dressing versus closed dressing in management of second-degree burn in children. Methods: A prospective comparative study was done on children ( $0-12$ years) presented with second-degree burn during the period of August 2009 to December 2010. More than 10\% total burn surface area (TBSA ) involving any region of the body and any percentage of TBSA involving the hands, feet, face, and perineum were included. Patients arrived only after 24 hours after burn; burn with other systemic illnesses were excluded. By randomized controlled trial (RCT) sampling technique, study population was divided into two groups-group A and group B. In group A, exposure or without dressing technique was followed and in group B, occlusive dressing technique was followed. Results: 125 patients were treated in each group. Age range was from 2 months 15 days to 10 years. The range of mean length of stay (LOS) in the hospital in group A was 5 to 26 days and in group B, 7 to 43 days. In group B, $42 \%$ (52) patients had wound infection, but in group A, it was only 15\%. Pseudomonas infection in group B was found in 40 cases. In group A it was only in 5 patients. In group B, 94\% patients needed cleaning and dressing under general anesthesia and in group A it was only $12 \%$. Eleven patients from group B needed skin grafting, but none from group A needed it. Mean total costs of management was USD 111.13 in group A, it was USD 182.85 in group B. Conclusion: The present study demonstrated that the open or exposure or without dressing method is suitable and effective in reducing patients' morbidity when compared to the closed or with occlusive dressing method for the management of second-degree burn wound in children. This method is also significantly cost effective.
\end{abstract}

Key words: Second-degree burn; Open dressing; Closed dressing.

\section{INTRODUCTION}

In terms of functional, social, and psychological impairment, the most devastating cause of childhood injury is burn. In the year of 2000, total number of global deaths due to burns was recorded as 23,800 ; the majority of which occurred in low and middle-income countries. ${ }^{1}$

In the developing countries, burn is almost certainly both greater and different in many respects, where paediatric burn comprises $45 \%$ of the total burn unit workload. ${ }^{2}$

\section{Dr. Kazi Md. Noor-ul Ferdous}

Department of Paediatric Surgery

Dhaka Shishu (Children) Hospital

Dhaka, Bangladesh

E-mail: kmnferdous@gmail.com 
In our country, burn was the fifth leading cause of child health illness. The incidence of burn is 288.1 per $1,00,000$ children per year and the morbidity is 0.6 per $1,00,000$ children. ${ }^{1}$ In the Department of Paediatric Surgery, Dhaka Shishu (Children) Hospital, burn is identified as the second most common surgical problem. ${ }^{3}$

First, the overall physiologic status of the child with burn should be assessed. As with trauma related injury, the primary and secondary survey are performed in Advanced Trauma Life Support (ATLS) standered. ${ }^{4}$ After the primary assessment, a burn specific secondary survey should be performed that includes a detailed assessment of the burn wounds and other injuries. $^{5}$

There are many methods of burn wound management, open or exposed, semi-closed or closed technique. With or without using topical antibiotics, has also been advocated. The open methods aim at drying up the burnt area as quickly as possible, leaving tissues to heal under a dry crust. The closed methods usually entail an initial debridement under anaesthesia or sedation, application of a topical antibiotic, and coverage with some form of sterile dressing. The dressing are changed daily or on regular interval, with or without sedation. ${ }^{6}$

From a practical point of view, the burn wound is better treated with dressing. Small children tend to prefer injured site to be covered with dressings. ${ }^{7}$

Methods for handling burn wounds have changed in recent decades. Traditional burn wound management involved applying topical antibiotic in dressing until the eschar separated. ${ }^{8}$ Dressings for patients with burns can be opened or closed. The open dressing reduces healing time and re-epithelialization time and promotes early elimination of crusts. However, open dressing makes it more difficult for patients with large burned areas to move. The occlusive dressing allows the patients to move freely. ${ }^{9}$ Total management cost is very high as it depends on the length of stay and demands multidisciplinary teams involving nurses, auxiliaries, surgeons, intensivists, psychologists, psychiatrists, nutritionists, and physiotherapists. Local care of burn wounds continues to account for a large proportion of the cost per day for treating patients with burns. Dressing costs, however, can vary according to the size and depth of the burned area, the type of dressing used, and the occurrence of complications. ${ }^{9}$ Like other countries, most of our burn unit believe the closed technique to be ideal for management of second-degree burn wounds. But Gosselin and Kuppers ${ }^{6}$ found the open or exposure technique to be better for the resource-poor, low-income developing countries e.g. Siera Leon, in terms of reduced morbidity, reduced length of hospital stay, and reduced treatment cost. ${ }^{6}$ There is no such study regarding the management of burn wounds without dressing in our country. So our study is intended to reduce the complications and morbidity related to burn and its treatment, the number of subsequent operative procedures needed for these patients, the length of hospital stay, and the burden of treatment cost upon the patient's family in our country.

\section{MATERIALS AND METHODS}

This prospective comparative study was conducted for a period of 16 months from August 2009 to December 2010 in the burn and plastic reconstructive unit of the Department of Paediatric Surgery, Dhaka Shishu (Children) Hospital and prior to that Institutional Review Board (IRE) approved the protocol.

All patients of paediatric age group ( $0-12$ years) admitted with second-degree burn, $>10 \%$ TBSA (total body surface area) involving any region of the body and any percentage TBSA involving the hands, feet, face, and perineum were included in this study.

Patients with second-degree burn, arrived only 24 hours after burn; burn with other systemic illness like protein energy malnutrition, were excluded.

Randomized controlled trial (RCT) sampling technique was followed to select the groups.

In group $\mathrm{A}$, exposure or without dressing technique and in group $\mathrm{B}$, occlusive dressing technique was followed.

\section{Management Protocol}

All the patients were resuscitated according to the standard Advanced Trauma Life Support (ATLS) protocol, if needed. Percentage of TBSA of burn was calculated according to "Lund \& Browder" chart and recorded on a pre-prepared chart for each patient.

Fluid resuscitation was done by using ringers lactate solution where the volume was calculated by using the "Parkland formula" ( $4 \times \%$ of burn $\times$ body weight in $\mathrm{kg}$ ). Depth of burn estimation was done clinically. Erythematous and painful burn wound that is covered with blisters is defined as seconddegree superficial burn.

All the patients were treated with prophylactic intra venous broad spectrum antibiotic (Inj. Cephradine, Inj. Flucloxacin and Inj. Amikacin) according to per kg body weight. Antibiotics were changed according to wound swab culture sensitivity reports. 
Initial analgesia was provided by injectable narcotic analgesic (Pethidine) according to per kg body weight, which was converted to Diclofenac sodium suppository and oral Paracetamol. Antihistamine (Promethazine $\mathrm{HCl}$ ) in oral form was advised to all patients as a sedative and as an antiitching agent. $\mathrm{H}_{2}$ blocker (Ranitidine) in the oral form according to per kg body weight was advised to all patients.

Wound swab was taken on the $3^{\text {rd }}$ day after admission for culture and sensitivity test in all patients.

In Group A: Patients were treated by initial cleansing of the burn surface by normal saline $(0.9 \% \mathrm{NaCl})$ under proper aseptic precautions. Application of tropical antiseptic ointment (2\% Silver sulphadiazine) was done all over the burn surface area. No occlusive dressing was applied, keeping the wound uncovered or exposed. Patients were advised to take daily bath using warm water followed by cleansing of the burn surface by normal saline $(0.9 \% \mathrm{NaCl})$ and application of $2 \%$ Silver sulphadiazine cream again.

Close follow up and inspection of the wound were done and signs of wound infection (discharge, foul smell, pyrexia) were looked for.

Cleaning wound under general anaesthesia was done if there was any of presence of purulent exudates or presence of devitalized tissue which could not be removed without surgical excision regular bath or persistent pyrexia.

In Group B: Patients were treated by initial cleansing of the burn surface by normal saline $(0.9 \% \mathrm{NaCl})$ under proper aseptic precautions. Application of tropical antiseptic cream (2\% Silver sulphadiazine) was done all over the burn surface area. The wound was covered with sterile dressing having three layers e.g., medicated tulle, povidone iodine soaked gauzes, cotton and dry gauze; and finally was wrapped by sterile bandage.

Close follow up and inspection of the wound covered by dressing was done. Decision of wound debridement and dressing change was taken on the basis of the following criteria $^{2}$ - when the integrity of the dressing was lost (strike through), soaked by exudates, presence of devitalized tissue could not be removed without surgical excision, persistent unexplained pyrexia, foul smell from dressing and positive wound swab culture.

All the patients were discharged form hospital when the wound healed up, and when no pyrexia was noted. and there was no need for further procedure.

The following outcome variables were studied, presence of wound infection (presence of bacterial colony on wound swab culture), cleaning and dressing under general anaesthe- sia, length of stay (LOS) in the hospital, average cost of treatment per (e.g., admission fees, bed rent, operation charge, cost of various pathological investigations, cost of intravenous fluids, normal saline, antiseptic cream, drugs, medicated tulle, other dressing related materials etc.).

\section{Data Calculation and Analysis}

All data were collected in data sheets by taking proper history, clinical examination, daily follow up, investigations e.g., culture sensitivity tests, keeping records of purchases of drugs and other costs. Collected data were arranged in systemic manner, presented in various tables and figures and statistical analysis was made to evaluate the objectives of the study. $p$ value $<0.05$ was taken as significant.

\section{RESULTS}

A total number of 12,144 patients were admitted to the Department of Surgery of Dhaka Shishu (Children) Hospital during the study period of 5 years. Out of them 813 patients were due to burn. From the admitted burned patients, 250 cases of second-degree superficial burn (scald) patients were selected as sample for the study.

The age range of the patient in this study was from 2 months 15 days to10 years. Age distribution of each group is given in Figure 1.

In group $A, 66 \%$ patients were male and $34 \%$ patients were female. In group B 55\% patients were male and 45\% patients were female.

Hot liquids (water, tea, milk, dal, rice water, etc) and flame were the main burning agents. Distribution of patients according to the causative agents and percentage of TBSA are shown in Tables 1 and 2.

Mean length of stay (LOS) in the hospital in group A, $13.8 \pm 4.62$ days (range: 5 to 26 days) and in group B,

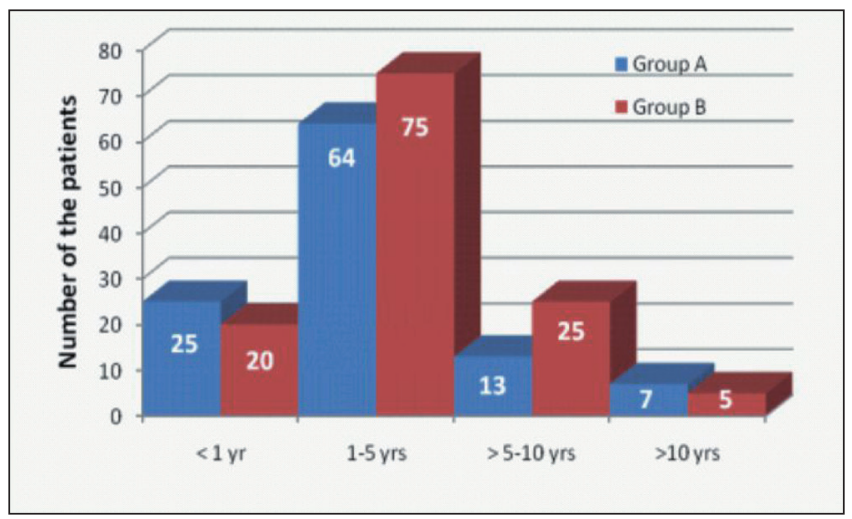

Figure 1: Age distribution of the patients 
Table 1: Patients according to the causative agents

\begin{tabular}{lcc}
$\begin{array}{c}\text { Cause of burn } \\
\text { injury }\end{array}$ & $\begin{array}{c}\text { Group A }(\mathbf{N}=125) \\
\text { Hot water }\end{array}$ & $\begin{array}{c}\text { Group B }(\mathbf{N}=125) \\
\text { Hot liquids (tea/ } \\
\text { milk/dal/rice }\end{array}$ \\
$\begin{array}{l}\text { water) } \\
\text { Hot vegetables/ }\end{array}$ & $22(17.6 \%)$ & $19(15.2 \%)$ \\
$\begin{array}{l}\text { curry/shuji } \\
\text { Flame }\end{array}$ & $10(8 \%)$ & $35(28 \%)$ \\
\hline
\end{tabular}

Table 2: Patients according to the percentage of TBSA of burn

\begin{tabular}{lcc} 
TBSA $(\%)$ & Group A $(\mathbf{N}=125)$ & Group B $(\mathbf{N}=125)$ \\
Up to 10\% & $50(40 \%)$ & $46(36.8 \%)$ \\
$11-15 \%$ & $47(37.6 \%)$ & $49(39.2 \%)$ \\
$16-20 \%$ & $16(12.8 \%)$ & $21(16.8 \%)$ \\
$>20 \%$ & $12(9.6 \%)$ & $9(7.2 \%)$ \\
Total & 125 & 125 \\
\hline
\end{tabular}

Table 3: Types of isolated organisms

\begin{tabular}{|c|c|c|}
\hline Types of organisms & $\begin{array}{l}\text { Group A } \\
(N=23)\end{array}$ & $\begin{array}{l}\text { Group B } \\
(N=50)\end{array}$ \\
\hline S. aureus & $13(56 \%)$ & $21(42 \%)$ \\
\hline S. aureus + Pseudomonas & - & $10(20 \%)$ \\
\hline \multicolumn{3}{|l|}{ auroginosa } \\
\hline E. coli & $5(22 \%)$ & $5(10 \%)$ \\
\hline S. aureus + E. coli & $0(00 \%)$ & $9(18 \%)$ \\
\hline P. auroginosa + E. coli & $5(22 \%)$ & $10(20 \%)$ \\
\hline
\end{tabular}

$17.8 \pm 6.38$ days (range: 7 to 43 days). The length of stay is significantly low in group A (Figure 2). ANOVA (Analysis of Variance) was done and $p$ value was 0.0042 , significant.

A significant difference in the rate of infection was observed between the two groups. In group A, only 15\% (19) patients had wound infection (positive wound swab culture) but in group B, 42\% (52) patients had wound infection. $\chi^{2}$ test done, $p$-value was 0.045 , which is significant.

Twenty-three patients in group $\mathrm{A}$ and 50 patients in group $B$, found positive culture of pathogenic organisms (Table 4$) \cdot \chi^{2}$ test done, $p$-value is significant (0.04). Culture sensitivity reports revealed that Staphylococcus aureus was sensitive to Flucloxacillin, Cephradine, and Cftriaxone antibiotic. Pseudomonas was sensitive to Gentamycin, Ceftazi- dime and Escherichia coli was sensitive to Gentamycin and Imipenum antibiotics. Infection was treated by using these sensitive antibiotics.

The types of isolated organisms in both groups are shown in Table 3.

Only 15 (12\%) patients in group A, needed cleaning the wound under general anaesthesia. Also the number of procedures was less than five in group A patients. In group B, 94\% patients (118) needed cleaning and dressing under general anaesthesia and maximum of them needed dressing more than five times. This is highly significant ( $\chi^{2}$ tests done, $p=$ 0.001).

Eleven patients of group B needed skin grafting, but none of group A needed it.

Mean cost of management (in US dollar, USD) per patient was calculated under several categories and finally total cost per patient was also calculated (Table 4$)$. Unpaired " $t$ " test done, $p$-value was highly significant (0.001).

\section{DISCUSSION}

The main goal of the management of acute burn injury in children is to save the life by prompt resuscitation which is followed by planned strategy to reduce the possible immediate and early morbidity. Management of burn wound is a vital issue and there are various techniques available for wound management. But, none of those has proved to be universally applicable. More and more new techniques are being proposed. Besides this fact, the cost effectiveness has also become an important factor for overall management of burned patients, practically in the developing countries.

In the present study, the length of stay in hospital is significantly less in patients treated without dressing or open or exposure method in comparison to control occlu-

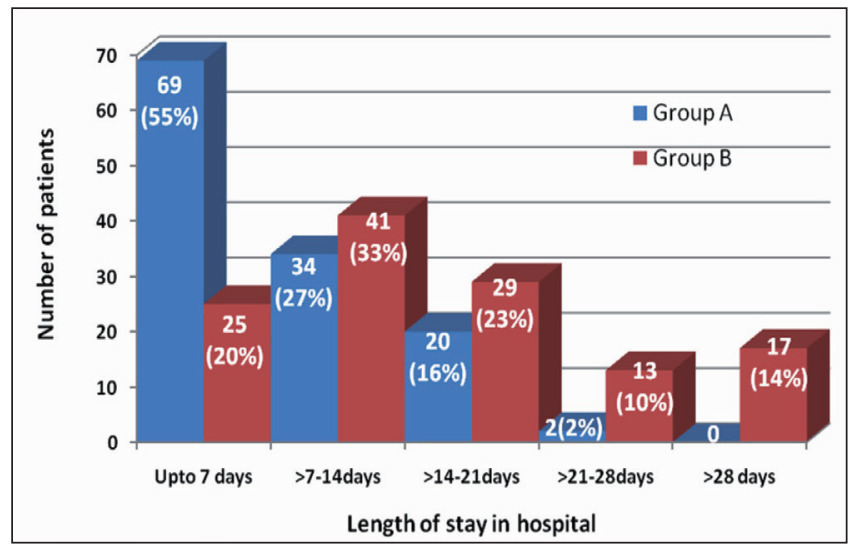

Figure 2: Comparison of length of hospital stay 
Table 4: Mean cost (USD) of management per patient between two groups

\begin{tabular}{lcc}
\multicolumn{1}{c}{ Category of cost } & Group A & Group B \\
Admission fee \& seat rent & $45.3 \pm 23.84$ & $56.62 \pm 2034.81$ \\
Investigations & $16.93 \pm 6.05$ & $14.06 \pm 3.12$ \\
Drugs & $43.170 \pm 16.23$ & $42.47 \pm 16.54$ \\
Dressing materials & $6.14 \pm 2.63$ & $12.14 \pm 6.92$ \\
Anaesthasia charge & $36.58 \pm 14.93$ & $59.14 \pm 19.62$ \\
Total cost & $111.13 \pm 49.04$ & $182.85 \pm 69.18$ \\
\hline
\end{tabular}

sive dressing method. The result is similar to that other studies. ${ }^{6,8}$ Atiyeh et al. ${ }^{8}$ used a different topical antiseptic cream (MEMO) keeping the patient exposed without applying any occlussive dressing. ${ }^{8}$ The reduced length of hospital stay depends on the depth of burn, \% TBSA, presence or absence of wound infection, number of operative procedure done etc.

In the present study, wound infection rate and less number of procedures under general anaesthesia needed in patients treated without dressing is compared to the group of patients treated with dressing. These factors possibly played an important role for less hospital stay in group A patients.

In this study, wound infection rate was significantly lower in patients treated without dressing than that of patients treated with occlusive dressing. Gosselin and Kuppers $^{6}$ also reported significant reduction in wound infection rate in patients treated with open technique, which is similar to our present study. However that study did not mention the type of microorganisms isolated on culture. In this study, the dressing was not changed on daily basis rather than changed according to the criteria mentioned by Lawrence, ${ }^{2}$ e.g., loss of integrity of dressing, soaked by exudates, foul smelling, pyrexia etc. Sometimes it was changed in between days when the children made the dressing dirty or made wet by water. Sometimes dressing applied close to thigh, legs, buttocks, or perineum became contaminated with stool or urine. Also there is a possibility of delay in changing the apparently contaminated or dirty dressing on time. All these factors possibly allowed the bacterial flora to multiply within the occlusive dressing. However, lack of proper aseptic precaution and improper sterilization of surgical equipments used for dressing may also be responsible for high rate of wound infection in group B patients. On the other hand, patients treated without dressing the wound was cleaned with antiseptic and normal saline followed by application of antiseptic cream. The technique was so simple and easy to perform which could be done by any healthcare person or even by the parents. If the wound is contaminated with dirt, stool or urine, it could be recognized immediately, and cleaned followed by application of cream. So in group A patients, there was no chance of delay in cleansing the dirt or contamination. The above mentioned factors may be responsible for the reduce rate of wound infection in group A patients.

The number of procedures under general anaesthesia was also significantly less in patients of group A in comparison to group B in our study. Gosselin and Kuppers ${ }^{6}$ have also shown similar results. Dressing done under anaesthesia reduces pain, distress, physiological and physiological trauma. It is also convenient to the doctors. General anaesthesia is considered for paediatric patients in early stage of admission. ${ }^{10}$ In this study, first and early 3-5 dressings were done under general anaesthesia. So in group B almost all patients required general anaesthesia. Eleven patients in group B, needed skin graft due to conversion of a superficial wound to a deep one. Only 15 patients of group A needed debridement under general anaesthesia and some light dressing due to wound infection, which was significant $(p=0.023)$.

The most difficult part of the study was to calculate the cost of overall management of burned patients in our hospital setup. There was significantly less amount of cost in group A compared to group B. Gosselin and Kuppers ${ }^{6}$ found that the cost of supply (dressing, sedative, antibiotic) was $2-3$ times less compared to open or exposed wound with an equal percentage of TBSA. The finding is almost similar in our study. Atiyeh et al. ${ }^{8}$ found that the use of moist exposed burn ointment (MEBO) without any dressing resulted in statistically very significant decrease in overall direct treatment cost. MEBO application also reduces the cost. ${ }^{8}$ Open method of burn management reduced length of hospital stay, reduced wound infection, reduced need of debridement under general anaesthesia, and no dressing change was required. These factors ultimately reduced the cost in group A than in group B patients.

\section{CONCLUSION}

The open or exposed or without dressing method for management of second-degree burn is better than closed or occlusive dressing method in limited resources developing country. Length of stay (LOS), wound infection rate, and procedure under general anaesthesia are significantly 
reduced in this method. This method does not need the painful dressing changing procedure, thus reducing the psychological trauma to children and their parents, saved the children from the hazards of repeated exposure to anaesthet- ic drugs, and reduced the cost of total burn wound management. The regular cleaning and cream application can easily be taught to the parents, so that the workload on the doctors and other hospital stuffs can be reduced significantly.

\section{REFERENCES}

1. Mashreky SR, Rahman A, Chowdhury SM, Giashuddin S, Svanstrom L, Linan M, Shafinaz S, Uhaa IJ, Rahman F. Consequences of childhood burn: findings from the largest community-based injury survey in Bangladesh. Burns 2008;34:91218.

2. Lawrence JC. Burns and scalds: etiology and prevention. In: Settle AD, editor. Principles and practice of burn management. $1^{\text {st }}$ ed. Edinburgh: Churchill Livingstone; 1996. pp. 3-13.

3. Masood AFM, Khan AR, Islam KM. Surgical problems I children - 10 years survey in Dhaka Shishu (Children) Hospital. Dhaka Shishu (Children) Hospital Journal 1991;7:24-29.

4. Duffy BJ, McLaughin PM, Eichelberger MR. Assessment, triage, and early management of burns in children. Clinical Pediatric Emergency Medicine 2006;7:82-93.

5. Shukla PC, Sheridan RL. Initial evaluation and management of burn patients. 2003. EMedicine.com Available at: www. Emedicine.com.

6. Gosselin RA, Kuppers B. Open versus closed management of burn wounds in a low-income developing country. Burns 2008;3: 644-47.

7. Carolyn R. Pediatric Burn. In: Settle AD, editor. Principles and practice of burn management. $1^{\text {st }}$ ed. Edinburgh: Churchill Livingstone; 1996. 377-79.

8. Atiyeh BS, Dham R, Kadry M, Abdullah AF, Al-Oteify M, Fathi O, Samir A. Benefit-cost analysis of moist exposed burn ointment. Burns 2002;28:659-69.

9. Torrati FG, Rossi LA, Ferreira E, Dalri MCB, Carvalho EC, Barberia CBS. Analysis of cost of dressings in the care of burn patients. Burns 2000;26:289-91.

10. Simons MA, Kimble RM. Pediatric Burn. In JH Stone, M Blouin editors. International Encyclopedia of Rehabilitation 2011. Available online: http://cirrie.buffalo.edu/encyclopedia/en/article/119/ 